\title{
THE TREATMENT OF LEPROMATOUS LEPROSY WITH NEOVADRINE AND VADRINE IN COMBINATION WITH DDS
}

\author{
By J. A. Allan, M.B., Ch.B. \\ Medical Superintendent, Ngomahuru Leprosy Hospital, \\ Fort Victoria, S. Rhodesia
}

Neovadrine and Vadrine are related compounds. The former is 2-pyridyl-(4)-1, 3, 4,-oxydiazolone-(5) and Vadrine is the p-aminosalicylate.

For the trial we chose 21 lepromatous cases without borderline features, and gave to 10 Neovadrine combined with DDS and to 11 Vadrine combined with DDS. Previous treatment in 15 cases had been DDS alone for varying periods. Biopsies were taken of all cases at the beginning of the trial, and biopsies were again taken 6 months later in order to establish the Biopsy Index and percentage fall for the period. During the trial clinical and bacterial examinations were made at intervals of 3 months, the first assessment at 9 months, and the second 3 months later. Results were compared with those of DDS. Dosage of Neovadrine and Vadrine began with 1 tablet daily of $200 \mathrm{mg}$. increasing by 1 tablet weekly to a maximum of $20 \mathrm{mg}$. per $\mathrm{kg}$. of body weight and $40 \mathrm{mg}$. $/ \mathrm{kg}$. per day, respectively. This combined with $100 \mathrm{mg}$. DDS daily.

\section{Conclusions}

Evaluation of the efficacy of Neovadrine/DDS and Vadrine/DDS compared with DDS, depends on clinical impressions and bàcteriological studies. These methods leave much to be desired, but are sufficient to formulate an opinion, whether, or not, the combinations, by virtue of increased effect, should replace DDS.

The yardstick of comparison is based on a study of a large number of lepromatous cases treated with DDS over the past 10 years.

The clinical impression gained, in this small series of cases, after 21 months of treatment, suggests a slightly better response, and manifested equally by both combinations. Cases Nos. 12 and 17 show an accelerated response. This phenomenon, however, can also occur with DDS, no doubt the result of mutation of immunological response in some individuals.

Examination of the Biopsy Index shows a mean percentage fall of $41 \%$ in six months, which compares favourably with results from elsewhere (RIDLEY). Unfortunately no figures are available for our own DDS cases. 
TABLE OF RESUltS

\begin{tabular}{|c|c|c|c|c|c|c|c|c|c|c|c|c|c|c|c|c|c|c|c|c|c|c|c|c|}
\hline \multicolumn{2}{|c|}{ Case No. } & \multicolumn{2}{|c|}{ Sex Age } & 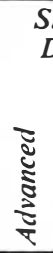 & $\begin{array}{l}\dot{\Sigma} \\
\dot{\Sigma} \\
\grave{\Sigma}\end{array}$ & 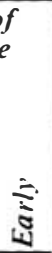 & $\begin{array}{l}\text { Previous } \\
\text { treatment } \\
\text { with } D D S\end{array}$ & $\begin{array}{l}21 \text { months of } \\
\text { combined } \\
\text { treatment } \\
\text { Neo/V|DDS } \\
\text { or } V \mid D D S\end{array}$ & 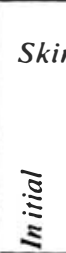 & $\begin{array}{c}n \text { sme } \\
\dot{亠} \\
\vdots \\
\vdots \\
\bar{\Xi} \\
\bar{\leftarrow}\end{array}$ & $\begin{array}{l}\text { Bact } \\
\text { ars }\end{array}$ & $\begin{array}{l}\text { teriolo } \\
\mathrm{N}\end{array}$ & $\begin{array}{l}g y \\
\text { asal sm } \\
\dot{\vdots} \\
\vdots \\
\vdots \\
\vdots \\
\bar{\nabla}\end{array}$ & $\frac{\overline{3}}{3}$ & $\begin{array}{l}\text { : } \\
: \\
\Xi\end{array}$ & $\begin{array}{l}\stackrel{\vdots}{\Xi} \\
\vdots \\
\vdots \\
\bar{\Xi}\end{array}$ & $\begin{array}{l}\text { idex } \\
\equiv \\
\approx\end{array}$ & \multicolumn{2}{|c|}{ 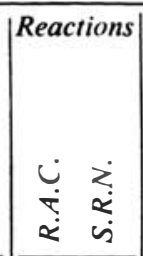 } & 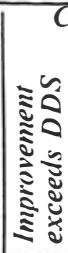 & 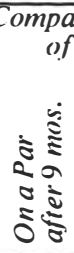 & 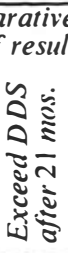 & 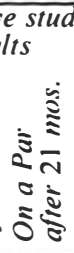 & 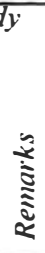 \\
\hline 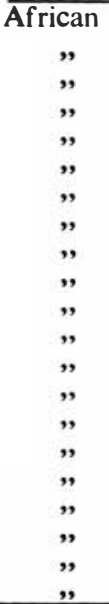 & $\begin{array}{r}1 \\
2 \\
3 \\
4 \\
5 \\
6 \\
7 \\
8 \\
9 \\
10 \\
11 \\
12 \\
13 \\
14 \\
15 \\
16 \\
17 \\
18 \\
19 \\
20 \\
21\end{array}$ & \begin{tabular}{l|}
$M$ \\
$M$ \\
$M$ \\
$M$ \\
$M$ \\
$M$ \\
$M$ \\
$M$ \\
$F$ \\
$M$ \\
$M$ \\
$M$ \\
$F$ \\
$F$ \\
$F$ \\
$F$ \\
$F$ \\
$M$ \\
$M$ \\
$M$
\end{tabular} & $\begin{array}{l}28 \\
30 \\
19 \\
20 \\
16 \\
42 \\
14 \\
38 \\
24 \\
45 \\
35 \\
45 \\
34 \\
20 \\
22 \\
36 \\
40 \\
35 \\
15 \\
34\end{array}$ & + & $\begin{array}{l}+ \\
+ \\
+ \\
+ \\
+ \\
+ \\
+ \\
+ \\
+ \\
+ \\
+ \\
+\end{array}$ & $\begin{array}{l}+ \\
+\end{array}$ & $\begin{array}{l}6 \text { mos. } \\
6 \text { mos. } \\
4 \text { mos. } \\
5 \text { mos. } \\
5 \text { mos. } \\
4 \text { mos. } \\
4 \text { mos. } \\
3 \text { mos. } \\
\text { Nil } \\
\text { Nil } \\
2 \text { mos. } \\
2 \text { mos. } \\
\text { Nil } \\
6 \text { mos. } \\
5 \text { mos. } \\
5 \text { mos. } \\
4 \text { mos. } \\
5 \text { mos. } \\
\text { Nil } \\
\text { Nil } \\
\text { Nil }\end{array}$ & $\begin{array}{l}\text { NeoV/DDS } \\
\text { NeoV/DDS } \\
\text { NeoV/DDS } \\
\text { NeoV/DDS } \\
\text { NeoV/DDS } \\
\text { NeoV/DDS } \\
\text { NeoV/DDS } \\
\text { NeoV/DDS } \\
\text { NeoV/DDS } \\
\text { NeoV/DDS } \\
\text { V/DDS } \\
\text { V/DDS } \\
\text { V/DDS } \\
\text { V/DDS } \\
\text { V/DDS } \\
\text { V/DDS } \\
\text { V/DDS } \\
\text { V/DDS } \\
\text { V/DDS } 11 \mathrm{mos} . \\
\text { V/DDS } \\
\text { V/DDS }\end{array}$ & $\begin{array}{l}+5 \\
+5 \\
+5 \\
+5 \\
+5 \\
+5 \\
+5 \\
+5 \\
+5 \\
+5 \\
+5 \\
+5 \\
+5 \\
+5 \\
+5 \\
+5 \\
+5 \\
+5 \\
+5 \\
+5 \\
+5\end{array}$ & $\begin{array}{l}+5 \\
+5 \\
+5 \\
+5 \\
+5 \\
+1 \\
+5 \\
+5 \\
+5 \\
+5 \\
+2 \\
+2 \\
+4 \\
+5 \\
+3 \\
+5 \\
+5 \\
+5 \\
+5 \\
+5 \\
+5\end{array}$ & $\begin{array}{l}+5 \\
+5 \\
+5 \\
+5 \\
+5 \\
+3 \\
+5 \\
+5 \\
+5 \\
+5 \\
+4 \\
+1 \\
+5 \\
+5 \\
+5 \\
+5 \\
+2 \\
+5 \\
+5 \\
+4 \\
+5\end{array}$ & $\begin{array}{l}+5 \\
+5 \\
+5 \\
+5 \\
+5 \\
+5 \\
+3 \\
+5 \\
+5 \\
+5 \\
+5 \\
+5 \\
+2 \\
+5 \\
+5 \\
+5 \\
+5 \\
+1 \\
+5 \\
+5 \\
+5\end{array}$ & $\begin{array}{c}+1 \\
+1 \\
+1 \\
+1 \\
+3 \\
\text { Neg. } \\
+3 \\
+4 \\
+1 \\
+2 \\
\text { Neg. } \\
\text { Neg. } \\
\text { Neg. } \\
+3 \\
\text { Neg. } \\
+1 \\
\text { Neg. } \\
\text { Neg. } \\
+1 \\
+2 \\
\text { Neg. }\end{array}$ & $\begin{array}{c}\text { Neg. } \\
+1 \\
\text { Neg. } \\
\text { Neg. } \\
+2 \\
\text { Neg. } \\
+3 \\
\text { Neg. } \\
\text { Neg. } \\
+1 \\
\text { Neg. } \\
\text { Neg. } \\
+1 \\
\text { Neg. } \\
\text { Neg. } \\
+1 \\
\text { Neg. } \\
\text { Neg. } \\
\text { Neg. } \\
\text { Neg. }\end{array}$ & $\begin{array}{c}5 \\
4.5 \\
4.5 \\
4 \\
4 \\
1 \\
6 \\
4 \\
\overrightarrow{5.4} \\
.45 \\
.24 \\
.25 \\
.5 \\
3.6 \\
4 \\
.5 \\
1.8 \\
3 \\
.75 \\
5\end{array}$ & $\begin{array}{l}3.2 \\
2.8 \\
2.7 \\
1 \\
3.6 \\
0.3 \\
0.6 \\
1.7 \\
\overline{2.8} \\
.23 \\
.09 \\
.08 \\
.08 \\
2.3 \\
3 \\
1 \\
1.4 \\
.1 \\
2.8 \\
2.8\end{array}$ & \begin{tabular}{|c|}
36 \\
38 \\
40 \\
75 \\
10 \\
70 \\
90 \\
57 \\
748 \\
49 \\
62 \\
68 \\
84 \\
36 \\
25 \\
-50 \\
22 \\
97 \\
-73 \\
44
\end{tabular} & + & + & $\begin{array}{l}+ \\
+ \\
+ \\
+ \\
+ \\
+ \\
+ \\
+ \\
+\end{array}$ & \begin{tabular}{|l}
$=$ \\
$=$ \\
$=$ \\
patie \\
$=$
\end{tabular} & $\begin{array}{l}+ \\
+ \\
+ \\
+ \\
+ \\
+\end{array}$ & $\begin{array}{c}= \\
= \\
= \\
= \\
= \\
\text { scond } \\
=\end{array}$ & ed \\
\hline
\end{tabular}

R.A.C.-Reactionary Anaemia Complex. S.R.N.-Symptomless Reactionary Nodules. P_Pregnant. 
Cases 6,11,13 and 15 showed bacteriological worsening of the skin smears after an average interval of 12 months, suggesting the emergence of resistant strains, and further indicates that the paminosalicylate component of Vadrine, likewise, is unable to prevent this.

Of note is the absence of toxicity attributable to these compounds. The incidence of cases with symptomless reactionary nodules is not exceptional. Case No. 15 experienced a more severe reaction, probably the result of pregnancy.

The efficacy of the combinations suggests an initial augmented bacteriostatic effect, unfortunately shortlived, but nevertheless useful. The phase of gradual resorption of infiltrates and bacterial clearing continues, and depends to a large extent on the individual's varied tissue responses. A forecast of the ultimate time interval required for arrest of the disease, suggests that a slight reduction only, compared with DDS, can be expected. This hardly warrants their routine usage for the treatment of lepromatous leprosy.

\section{Summary}

The Oxydiazolones appear to be non-toxic when used in combination with DDS.

Their adjuvant action suggests an initial additive effect only, followed later by bacteriological worsening, suggesting the emergence of resistant organisms. The p. aminosalicylate component of Vadrine does not prevent this.

It is unlikely that the ultimate duration of treatmen $t$ required will be appreciably reduced compared with DDS.

\section{Acknowledgments}

I wish to express thanks to Ed. Geistlich Sons Ltd., Wolhusen, Switzerland, for their generous supplies of Neovadrine and Vadrine, and Drs. Jopling and Ridley for their co-operation and for undertaking the Biopsy Index studies.

Thanks are due to the Director of Medical Services, Southern Rhodesia, for permission to publish.

\section{Ref erence}

RiDLEY, D. S., (1958), Leprosy Rev., vol. 29, No. 1, p. 45. 